\title{
In-line evanescent-wave microfluidic absorption sensor based on an embedded optical microfiber coil
}

\author{
R. Lorenzi, ${ }^{1,2 *}$ Y. Jung, ${ }^{2}$ and G. Brambilla ${ }^{2}$ \\ ${ }^{I}$ Department of Materials Science, University of Milano-Bicocca, via R. Cozzi 53, 20125 Milano, Italy \\ ${ }^{2}$ ORC, University of Southampton, Southampton, SO17 1BJ, UK \\ *Corresponding author: roberto.lorenzi@mater.unimib.it
}

\begin{abstract}
We present the absorption spectra collected with an evanescent-field absorption sensor. The device comprises an active fluidic channel with an embedded microfiber coil resonator. Deviations from Beer-Lambert law will be discussed in terms of adsorption mechanism.

OCIS codes: 060.4005 Microstructured fibers, 160.6030 Silica, 280.4788 Optical sensing and sensors, 240.6700 Surfaces
\end{abstract}

Optical micro- and nano- fibers represent a useful tool for sensor technologies because of their large interactions between the evanescent component of the propagating wave (EW) and the surrounding medium. Here we present a novel design for EW absorption sensors based on microfiber coils. The main advantage of our approach is that the absorption measurement takes place in-situ while the analyte is flowing in the device, since the responsive coil resonator is buried in the fluidic channel walls. The device fabrication strategy comprises 4 steps (as shown in Fig. 1a): 1) tapering a standard optical fibre by the modified flame brush technique (final diameter $2.5 \mu \mathrm{m}$ ) [1], 2) coiling the tapered fibre onto an expendable support rod of PMMA that connects two Teflon tubes, 3) embedding the PMMA rod and the coil resonator with a Teflon solution, and 4) dissolving the support rod by dipping the device in acetone for 1 day. During the whole fabrication process the free spectral range, originated by the coil resonator, was monitored; ensuring that the optical path length of a single turn is not modified during device fabrication. The ends of the fluidic channel are connected by a pair of heat-shrink tubing to Teflon tubes and the liquid to be analysed is inserted with a syringe.
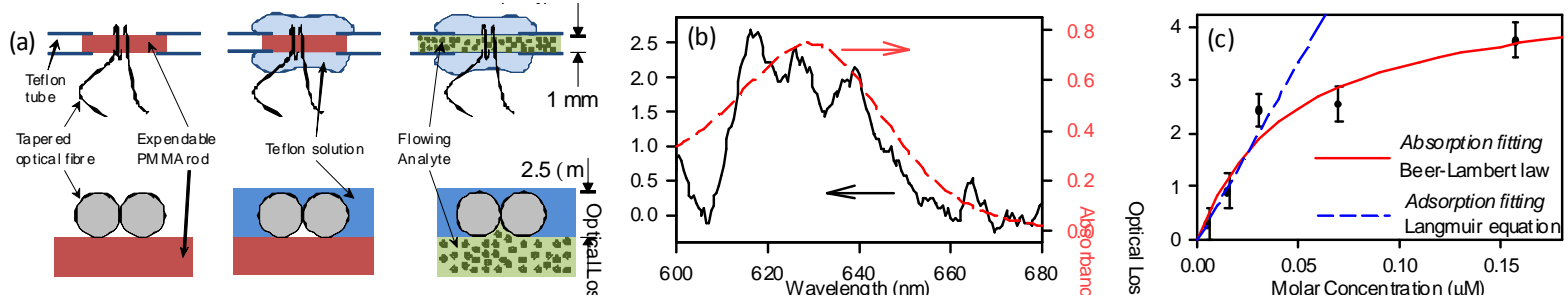

Fig. 1: (a) Schematic of the fabrication process, (b) Absoprtion spectrum of brilliant blue FCF recorded with the proposed device (solid black line) and with a spectrophotometer (dashed red line), and (c) Optical losses as a function of analyte concentration and fitting curves assuming EW absorption (dashed blue line) and adsorption mechanism (solid red line).

The device was tested measuring the optical absorption of aqueous solutions of Brilliant Blue FCF with concentration ranging from $6 \mathrm{mM}$ up to $0.18 \mathrm{mM}$. Optical spectra were recorded using a supercontinuum generator as light source and an OSA as detector. Absorption spectra recorded with the proposed device (Fig. 1b) show that results are in reasonable accordance with the reference spectrum, although measurements are strongly affected by the noise fluctuation of the source. Fig. 1c shows the optical losses as a function of the analyte concentration. Dashed blue line represents the expected optical loss calculated by a modified Beer-Lambert law adapted from the Gupta model of EW absorption in tapered fibres [2]. Within the experimental error the model predicts the observed optical losses for low analyte concentration. However for higher concentrations, experimental results show strong deviations, interpreted as a consequence of adsorption processes. Hence, optical losses have been fitted by means of Langmuir equation, thus assuming optical absorption proportional to the number of analyte molecules attached onto the fibre surface rather than the concentration of flowing analyte, as already observed in other EW absorption sensors [3]. The fitting result (red line in Fig. 1c) is in good agreement with the observed optical losses, within the complete concentration range.

\section{References:}

1. Y. Jung, G. S. Murugan, G. Brambilla, and D. J. Richardson, IEEE Photonic. Tech. L. 22, 1638 (2010).

2. B.D. Gupta and C.D. Singh, Applied Optics 33, 2737 (1993).

3. F. Warken, E. Vetsch, D. Meschede, M. Sokolowsi, and A. Rauschenbeutel, Opt. Express 15, 11952 (2007). 